\title{
CKAP2 phosphorylation by CDK1/cyclinB1 is crucial for maintaining centrosome integrity
}

\author{
Bum Ho Yoo ${ }^{1,5}$, Du-Seock Kang ${ }^{2,5}$, Chi-Hu Park ${ }^{3}$, Kyeongjin Kang ${ }^{4}$ and Chang-Dae Bae ${ }^{1}$ \\ Previously, we have reported that CKAP2 is involved in the maintenance of centrosome integrity, thus allowing for proper mitosis \\ in primary hepatocytes. To understand this biological process, we identified the mitosis-specific phosphorylation sites in mouse \\ CKAP2 and investigated CKAP's possible role in cell cycle progression. Because we observed mouse CKAP2 depletion in \\ amplified centrosomes and aberrant chromosomal segregation, which was rescued by ectopic expression of wild-type CKAP2, \\ we focused on the centrosome duplication process among the various aspects of the cell cycle. Among the identified \\ phosphorylation sites, T603 and possibly S608 were phosphorylated by CDK1-cyclin B1 during mitosis, and the ectopic \\ expression of both T603A and S608A mutants was unable to restore the centrosomal abnormalities in CKAP2-depleted cells. \\ These results indicated that the phosphorylation status of CKAP2 during mitosis is critical for controlling both centrosome \\ biogenesis and bipolar spindle formation.
}

Experimental \& Molecular Medicine (2017) 49, e354; doi:10.1038/emm.2017.92; published online 14 July 2017

\section{INTRODUCTION}

During every cell cycle, cells must accurately divide into two daughter cells with identical genetic information. If these processes are unregulated, daughter cells undergo tumor development through various mechanisms, including multipolar spindle formation and chromosome mis-segregation. ${ }^{1-5}$ For accurate division of the genetic material, the centrosome duplicates precisely once per cell cycle. The centrosome is a central organizer of cellular events, and it plays a crucial role in proper chromosome segregation during mitosis. ${ }^{6,7}$ Therefore, centrosome aberration serves as a hallmark of aggressive tumor and malignancy in clinical medicine. ${ }^{8}$

In our previous reports, we have shown that CKAP2, a cytoskeleton-associated protein, is essential for maintaining centrosome integrity and for chromosome segregation, thus maintaining genomic stability in primary hepatocytes. ${ }^{9}$ CKAP2 is mainly expressed in actively proliferating cells, including human cancer cells. ${ }^{10,11}$ The CKAP2 expression level changes in over the course of the cell cycle, ${ }^{12}$ and CKAP2 shows a distinct intracellular distribution during the cell cycle. ${ }^{10-12}$ The overexpression of CKAP2 induces microtubule bundling during interphase and formation of monopolar mitotic spindles during mitosis, as a result of a separation defect. ${ }^{13}$ The knockdown of CKAP2 induces retardation of cell proliferation, decreased cell viability, abnormal nuclear morphology, chromosome bridge formation and increased chromosomal instability. ${ }^{11}$ These findings suggest that CKAP2 may be involved in centrosome function and mitotic spindle dynamics, thus allowing for proper cell division.

However, how CKAP2 maintains the proper number of centrosomes for bipolar spindle formation is not known. Our previous report has shown that the mitosis-related functions of CKAP2 are regulated by phosphorylation. At least four different residues located near the C terminus (T578, T596, T622 and S627) of human CKAP2 play roles in mitosis. ${ }^{14}$ Among these phosphorylation sites, T622, which is phosphorylated by $\mathrm{CDK} 1 /$ cyclin $\mathrm{B} 1$, directly regulates spindle dynamics and proper bipolar spindle formation. ${ }^{14}$ In addition, S627, which is phosphorylated by Aurora B kinase, is required for the proper localization of CKAP2 at microtubules and centrosomes throughout mitosis. ${ }^{15}$ Therefore, we hypothesized that the phosphorylation status of CKAP2 might be related to centrosome integrity, and we investigated the mitosis-specific phosphorylation sites of mouse CKAP2 by using the BioID

\footnotetext{
${ }^{1}$ Department of Molecular Cell Biology and Samsung Biomedical Research Institute, School of Medicine, Sungkyunkwan University, Gyeonggi-do, Republic of Korea; ${ }^{2}$ Biomedical Research Center, Korea Advanced Institute of Science and Technology (KAIST), Daejeon, Republic of Korea; ${ }^{3}$ Research and Development Center, HuGeX Co. Ltd., Gyeonggi-do, Republic of Korea and ${ }^{4}$ Department of Anatomy and Cell Biology and Samsung Biomedical Research Institute, School of Medicine, Sungkyunkwan University, Gyeonggi-do, Republic of Korea

${ }^{5}$ These authors contributed equally to this work.

Correspondence: Professor C-D Bae, Department of Molecular Cell Biology and Samsung Biomedical Research Institute, School of Medicine, Sungkyunkwan University, 2066 Seobu-ro, Jangan-gu, Suwon-si, Gyeonggi-do 16419, Republic of Korea.

E-mail: cdbae@skku.edu
}

Received 24 January 2017; accepted 31 January 2017 
system, which is based on the biotinylation of proteins in the proximity of a BirA mutant in living cells. ${ }^{16}$ From these results, we elucidated the role of its phosphorylation status in centrosome integrity during mitosis.

\section{MATERIALS AND METHODS}

\section{Cell culture and cell cycle arrest}

NIH 3 T3 and HEK 293T cells obtained from ATCC (American Type Culture Collection, Manassas, VA, USA) were cultured in DMEM supplemented with $10 \%$ fetal bovine serum and $1 \%$ penicillinstreptomycin at $37^{\circ} \mathrm{C}$ in $5 \% \mathrm{CO}_{2}$. Cells were arrested in the $\mathrm{M}$ phase with $100 \mathrm{ng} \mathrm{ml}^{-1}$ of nocodazole (Sigma, St Louis, MO, USA) and $10 \mu \mathrm{M}$ of RO3306 (Enzo, Farmingdale, NY, USA) for $16 \mathrm{~h}$. HEK $293 \mathrm{~T}$ cells for lentiviral packaging were maintained in Opti-MEMGlutaMAX (Invitrogen, Carlsbad, CA, USA) supplemented with 5\% fetal bovine serum, $10 \mathrm{~mm}$ of sodium butyrate and $1 \%$ penicillinstreptomycin.

\section{Generation of a lentiviral vector construct}

Lentivirus particles were produced with Block-iT HiPerform Lentiviral Pol II miR RNAi Expression Systems (Invitrogen). Briefly, a doublestranded oligonucleotide encoding the UTR-targeting miR-CKAP2 (5'-TATGGTTGTGTTACTCCTGTC- $\left.3^{\prime}\right)$ was synthesized and cloned into pLenti6.4/R4R2/V5-DEST (Invitrogen). The CKAP2 cDNA or phosphorylation-deficient mutant cDNA was amplified by two-step PCR using a specific primer pair containing recombination sequences and was cloned into pLenti6.4/R4R2/V5-DEST (Invitrogen).

\section{Lentivirus production and transduction}

The lentiviral expression module was transfected into $2 \times 10^{6} \mathrm{HEK}$ 293T cells using PEI (Polysciences, Warrington, PA, USA), along with the helper plasmids pLP1, pLP2 and pLP/VSV-g (Invitrogen). The virus-containing supernatant was collected at both 2 and 3 days after transfection. The supernatant was filtered (pore size $0.45 \mu \mathrm{m}$ ) and concentrated using PEG-6000 (Sigma). The lentivirus particles were stored at $-80^{\circ} \mathrm{C}$ before use. For transduction, the lentivirus particles were supplemented with polybrene $\left(8 \mu \mathrm{g} \mathrm{ml}^{-1}\right.$, Santa Cruz Biotechnology, Santa Cruz, CA, USA), and then added to the cells for $8 \mathrm{~h}$.

\begin{abstract}
Affinity capture of biotinylated CKAP2
Biotinylated CKAP2 was purified by affinity capture, as described by Roux et al. ${ }^{16}$ Briefly, cells expressing the BirA-CKAP2 fusion protein were incubated for $24 \mathrm{~h}$ in complete medium supplemented with $50 \mu \mathrm{m}$ of biotin (Sigma). After being washed three times with PBS, the cells were lysed in $10 \mathrm{ml}$ of lysis buffer (50 mm Tris, $\mathrm{pH} 7.5,150 \mathrm{~mm}$ $\mathrm{NaCl}, 0.1 \%$ SDS, 1 mм EDTA, 1 mм EGTA, $1 \%$ Triton X-100, phosphatase inhibitor and complete protease inhibitor) at $4{ }^{\circ} \mathrm{C}$ for $1 \mathrm{~h}$. The sample was briefly sonicated and centrifuged at $16000 \mathrm{~g}$ at $4^{\circ}$ $\mathrm{C}$ for $30 \mathrm{~min}$. The supernatants were incubated with $200 \mu \mathrm{l}$ of Dynabeads (MyOne Steptavidin C1, Invitrogen) overnight at $4{ }^{\circ} \mathrm{C}$. The beads were collected and washed twice for $5 \mathrm{~min}$ in $2 \mathrm{ml}$ of lysis buffer and twice with $2 \mathrm{ml}$ of $50 \mathrm{~mm}$ ammonium bicarbonate ( $\mathrm{pH} 8.3$ ). Then, $10 \%$ of the magnetic beads bound to biotinylated proteins with $100 \mu \mathrm{l}$ of Laemmli SDS sample buffer were used for western blotting, and the remaining beads were analyzed by mass spectrometry.
\end{abstract}

\section{In vitro kinase assay}

Recombinant CDK1/cyclin B1 and Aurora B/INCENP were purchased from Carna Biosciences (Kobe, Japan), and recombinant histone H1 and kemptide were purchased from Merck Millipore (Billerica, MA,
USA). The kinase and substrate were incubated in kinase buffer (50 mм Tris, pH 7.5, $10 \mathrm{~mm} \mathrm{NaCl}, 10 \mathrm{~mm} \mathrm{MgCl}_{2}, 100 \mu \mathrm{g} \mathrm{ml}{ }^{-1} \mathrm{BSA}$, and $1 \mathrm{~mm}$ DTT) containing phosphatase inhibitor cocktail 1 (Sigma) and $50 \mathrm{~mm}$ ATP (Sigma) for $1 \mathrm{~h}$ at $30^{\circ} \mathrm{C}$. The level of unincorporated ATP was measured with Kinase-Glo Luminescent Kinase assays (Promega, Madison, WI, USA) using an Infinite M200 (Tecan, Männedorf, Switzerland). Synthetic peptides were produced at Peptron (Daejeon, Korea). The synthetic peptides used were as follows: T603: LKELKFLTPVRRSRR, T603A: LKELKFLAPVRRSRR, S608: FLTPVRRSRRIQDKT, S608A: FLTPVRRARRIQDKT.

\section{Mass spectrometry}

All nano-liquid chromatography-tandem mass spectrometry (LC-MS/MS) experiments were performed on an Ultimate 3000 system (Thermo Fisher Scientific, Bremen, Germany) connected to a mass spectrometer (HCTultra PTM Discovery System, Bruker Daltonics, Bremen, Germany) with a nanoelectrospray ion source. The tryptic digested peptides were separated on a $15-\mathrm{cm}$ analytical column (Zorbax 300SB C18, $0.075 \mathrm{~mm} \times 100 \mathrm{~mm}$, Agilent Technologies, Waldbronn, Germany) with a 90 -min gradient from 5 to $60 \%$ acetonitrile in $0.1 \%$ formic acid. The effluent from the nanoLC was directly electrosprayed into the mass spectrometer. The mass spectrometer was operated in auto-tandem mass spectrometry acquisition to automatically switch between full-scan MS and MS/MS acquisition. After acquisition of the MS spectra over the mass range for $\mathrm{m} / \mathrm{z}$ of $300-1600 \mathrm{Da}$, the three most intense peptide ions with charge states $\geqslant 2$ were sequentially isolated and fragmented in an ion trap by an alternative mode of collision-induced dissociation and electron transfer dissociation. MS/MS spectra were acquired over the mass range of $m / z 100-2800 \mathrm{Da}$. The voltage between the ion spray tip and the spray shield was set to $2.0 \mathrm{kV}$. Drying nitrogen gas was heated to $200{ }^{\circ} \mathrm{C}$, and the flow rate was $101 \mathrm{~min}^{-1}$. The collision energy was set automatically according to the mass and charge state of the peptides chosen for fragmentation.

All MS/MS spectra were identified by using the MASCOT v2.3 program (Matrix Science, London, UK), searching against the latest UniProt mouse database. To search for the phosphorylation sites of CKAP2, we used the following search parameters: maximum missing cleavage sites, 4; fixed modification, carbamidomethyl (C); variable modification, oxidation $(\mathrm{M})$, biotin $(\mathrm{K})$, biotin (N-term), phosphor (Y) and phosphor $(\mathrm{S}, \mathrm{T})$. The identified phosphorylation sites were inspected manually.

\section{Immunostaining}

Cells grown on sterile 22-mm coverslips were fixed in cold methanol for $30 \mathrm{~min}$ at $4{ }^{\circ} \mathrm{C}$ and blocked for $30 \mathrm{~min}$ in $3 \%$ BSA in PBST (phosphate-buffered saline, pH 7.5 with $0.05 \%$ Tween-20). The cells were then incubated for $2 \mathrm{~h}$ at room temperature with the primary antibody in blocking solution. Then, the cells were washed three times with PBST and incubated for $2 \mathrm{~h}$ at room temperature with anti-rabbit or anti-mouse secondary antibodies conjugated to Alexa Fluor 488 or Alexa Fluor 546 (1:5000, Invitrogen). The cells were subsequently stained with DAPI $\left(10 \mu \mathrm{g} \mathrm{ml}^{-1}\right)$. The primary antibodies were diluted as follows: mouse anti-CKAP2 (B-1-13) (1:1000, Hong et al. $\left.{ }^{11,14,17}\right)$, rabbit anti- $\gamma$-tubulin (1:2000, Abcam, Cambridge, MA, USA), mouse anti-myc (1:1000, Calbiotech, Spring Valley, CA, USA) and Alexa 546-conjugated streptavidin (1:1000, Abcam). The cells were mounted onto glass slides using Fluoromount (Sigma) for subsequent microscopic observation. 


\section{Western blotting}

Protein samples were separated by $8 \%$ SDS-polyacrylamide gel electrophoresis and electroblotted onto a polyvinylidene difluoride membrane (Merk Millipore). The membrane was blocked for $30 \mathrm{~min}$ in $5 \%$ skim milk in TBST (TBS, $0.1 \%$ Tween 20 ), incubated with the primary antibody in blocking solution overnight at $4{ }^{\circ} \mathrm{C}$, and washed three times with TBST. The membrane was then incubated with HRP-conjugated secondary antibodies for $1 \mathrm{~h}$ at room temperature and washed three times with TBST. The antibodies used were mouse anti-CKAP2 $\left(1: 3,000\right.$, Hong et al. $\left.{ }^{11,14,17}\right)$, rabbit anti- $\alpha$-tubulin (1:5000, Abcam), rabbit anti-CKAP2 pT603 (1:1000, Hong et al. ${ }^{14}$ ) and mouse anti-myc (1:1000, Calbiotech). Anti-mouse IgG, HRP-conjugated and anti-rabbit IgG, and HRP-conjugated antibodies (1:5000, Invitrogen) were used for secondary labeling. For the detection of signals, SuperSignal West Pico (Thermo Fisher Scientific, Rockford, IL, USA) was used according to the manufacturer's recommendations. Images were acquired using an LAS-3000 (Fujifilm, Tokyo, Japan) and Image Gauge software (Fujifilm, Tokyo, Japan).

\section{RESULTS}

\section{CDK1 inhibition triggers multiple centrosomes}

CDK1 inhibition triggers multiple centrosomes resulting from premature centriole disengagement because of prolonged G2/M arrest in HeLa cells. ${ }^{18,19}$ Thus, we hypothesized that CDK1 might phosphorylate the cognitive proteins in centrosomes, thus controlling centrosome number, and we confirmed this phenomenon in NIH 3T3 cells. To inhibit CDK1, NIH 3T3 cells were treated with the CDK1 inhibitor RO3306 for $16 \mathrm{~h}$. As a result, $\sim 25 \%$ of mitotic cells showed multiple centrosomes, whereas $\sim 1.5 \%$ of DMSO-treated cells showed multiple centrosomes (Figure 1). This result suggests that CDK1 might play some role in controlling the centrosome number by phosphorylating the cognitive proteins in centrosomes during early mitosis to maintain centrosome integrity.

\section{Identification of the phosphorylation sites of CKAP2}

To identify the phosphorylation sites of CKAP2 during the mitotic phase, we purified biotinylated CKAP2 using a BioID system. We produced the BirA-CKAP2 fusion protein and expressed it in NIH 3T3 cells using a lentivirus system.
As shown in Figure 2, BirA-CKAP2 was well expressed in our western blot samples (Figure 2a), and the protein showed the same localization on mitotic spindles and centrosomes as wild-type mouse CKAP2 after immunostaining for the Myc antibody (Figure 2b). In addition, in the immunostaining for streptavidin, biotinylated mouse CKAP2 exactly colocalized with the wild-type CKAP2 proteins. This result indicated that the BirA-CKAP2 protein behaved as the wild-type CKAP2 protein and the biotinylated protein.

To purify these proteins, we performed streptavidin affinity chromatography. The resulting proteins were separated by SDS-polyacrylamide gel electrophoresis and stained with Coomassie blue. As shown in Figure 2c, we obtained a large amount of biotinylated BirA-CKAP2, along with other biotinylated proteins. Then, the area of biotinylated BirA-CKAP2 was excised and digested by using sequencemodified trypsin. To enrich for the population of phosphopeptides from CKAP2, we performed $\mathrm{TiO}_{2}$ chromatography and then analyzed the samples by using nanoflow-liquid chromatography-mass spectrometry.

As a result, we identified six phosphorylation sites (Supplementary Figure 1c). Among them, three sites (S118, S216 and S261) were newly identified as phosphorylation sites. In addition, two sites (T561 and T579) have been reported as the mitosis-specific phosphorylation sites in human CKAP2. ${ }^{14}$

\section{In vitro phosphorylation assay}

In addition to T578 and T596, two additional sites (T622 and S627) have been reported as the mitotic phosphorylation sites of human CKAP2 in our previous reports. The amino-acid sequence around these two residues (T622 and S627) is highly conserved between human and mouse CKAP2 (Figure 2d). However, we were unable to identify these corresponding phosphorylation sites of mouse CKAP2 by using the BioID system. Therefore, we synthesized a peptide spanning this region around the two sites (T603 and S608), and we phosphorylated the synthetic peptide in vitro using the CDK1-cyclin B1 complex or Aurora B/INCENP (Supplementary Figure 1a and b), which is the kinase that
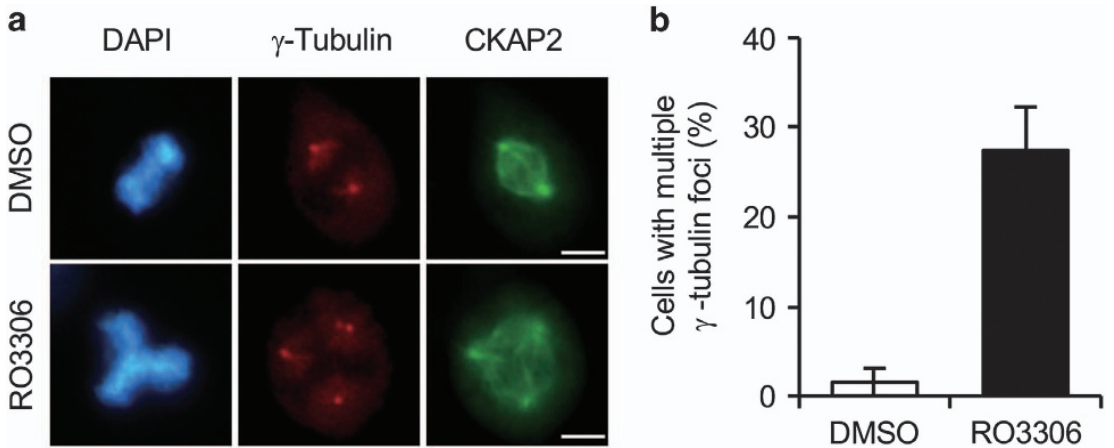

Figure 1 R03306 triggers multiple centrosomes. (a) NIH 3T3 cells treated with R03306 or DMSO for $16 \mathrm{~h}$ were co-immunostained for $\gamma$-tubulin (red), CKAP2 (green) and DAPI (blue). Representative images show multiple $\gamma$-tubulin foci in R03306-treated cells. Scale bar, $5 \mu \mathrm{m}$. (b) The quantification of cells with multiple $\gamma$-tubulin foci, presented as the mean values \pm s.e.m. 
phosphorylates human CKAP2. As a result, T603 and S608 were phosphorylated by the CDK1-cyclin B1 complex (Figures 2e and f). Therefore, we focused on these four phosphorylation sites (T561, T579, T603 and S608) and examined their roles in maintaining centrosome integrity.
T603 and S608 from CKAP2 are crucial for centrosome biogenesis

We have previously reported that multiple $\gamma$-tubulin foci with two centrioles triggered by CKAP2 depletion are a specific effect of CKAP2 depletion. ${ }^{9}$ Therefore, we investigated whether a

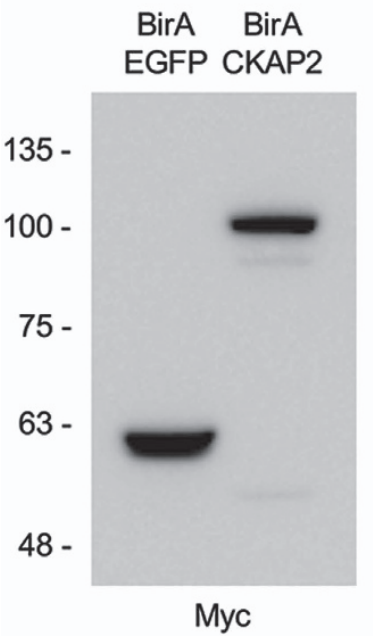

d

b

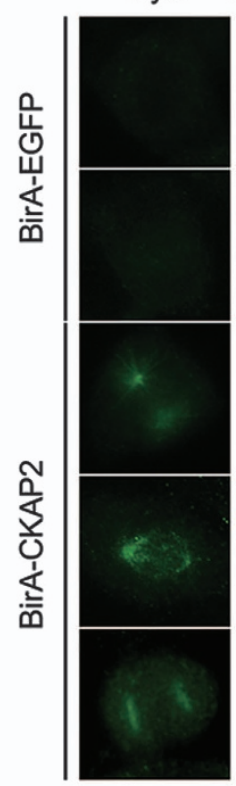

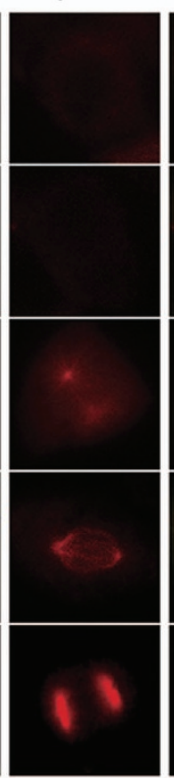

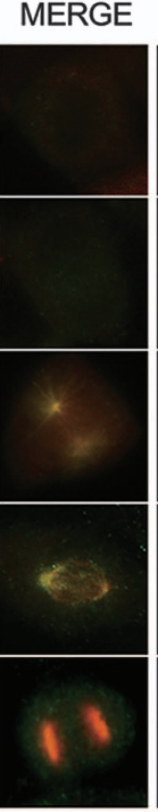

DAPI

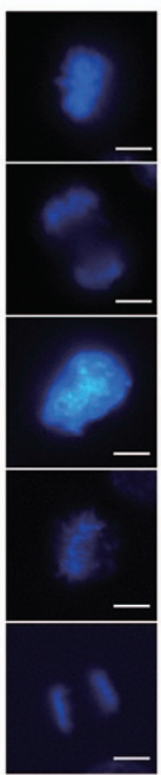

C

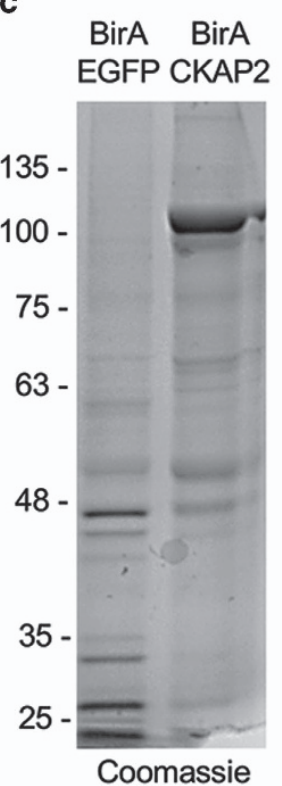

561

579

603608

Mouse 550 DNKIKDPD--LTTPDSKTEAGCIIRYNVSSTPRLQSMKKMQHDK-NSTLKELKFLTPVRRSRRIQ 623

Human 566 DNKTKDPTHDVKTPNTETRTSCLIKYNVSTTPYLQSVKKKVQFDGTNSAFKELKFLTPVRRSRRLQ 631

578

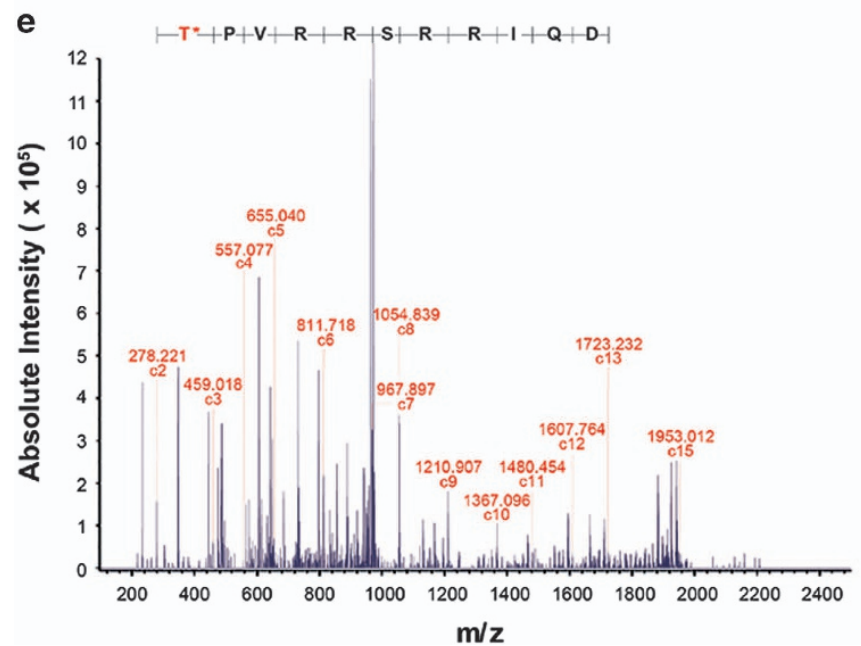

622627

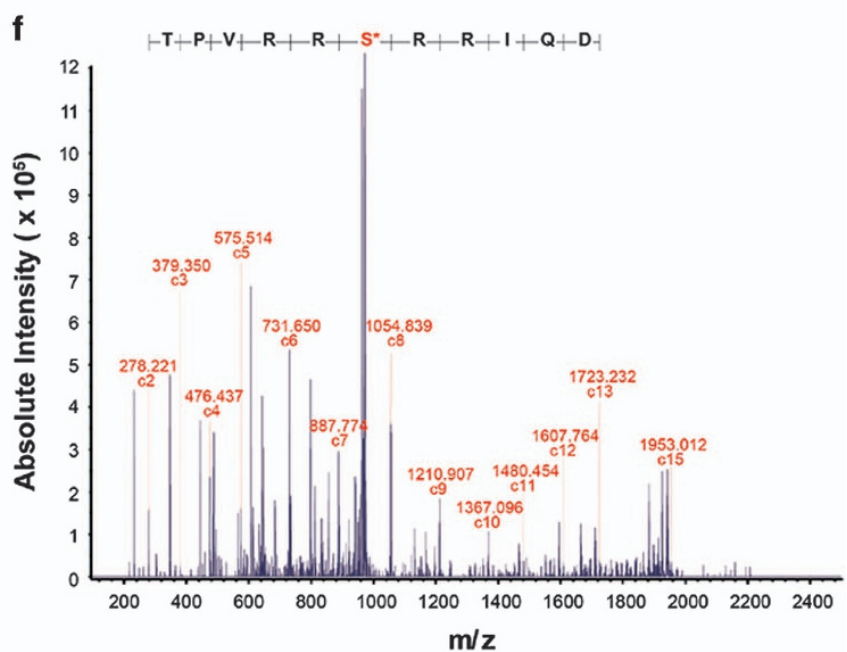

Figure 2 The localization and purification of BirA-CKAP2 and its phosphorylation sites. (a) The expression of BirA-EGFP or BirA-CKAP2 was confirmed by western blot analysis with anti-Myc antibody. (b) The ectopic expression of BirA-EGFP or BirA-CKAP2 in NIH 3T3 cells that were co-immunostained with streptavidin (red) and myc (green) and merged with DAPI (blue) after treatment with biotin. Representative images show that biotinylated CKAP2 localized to spindles, centrosomes and chromosome. Scale bar, $5 \mu \mathrm{m}$. (c) Biotinylated proteins purified using affinity purification with streptavidin and visualized by Coomassie staining. (d) The human and mouse homologs of CKAP2 were compared in terms of their amino-acid sequences with the identified phosphorylated sites in humans and the corresponding sites in mice (red). (e, f) The peptides including T603 and S608 were phosphorylated by the CDK1/cyclin B1 complex in vitro and were analyzed using ETD mass spectrometry. ETD, electron transfer dissociation. 
a
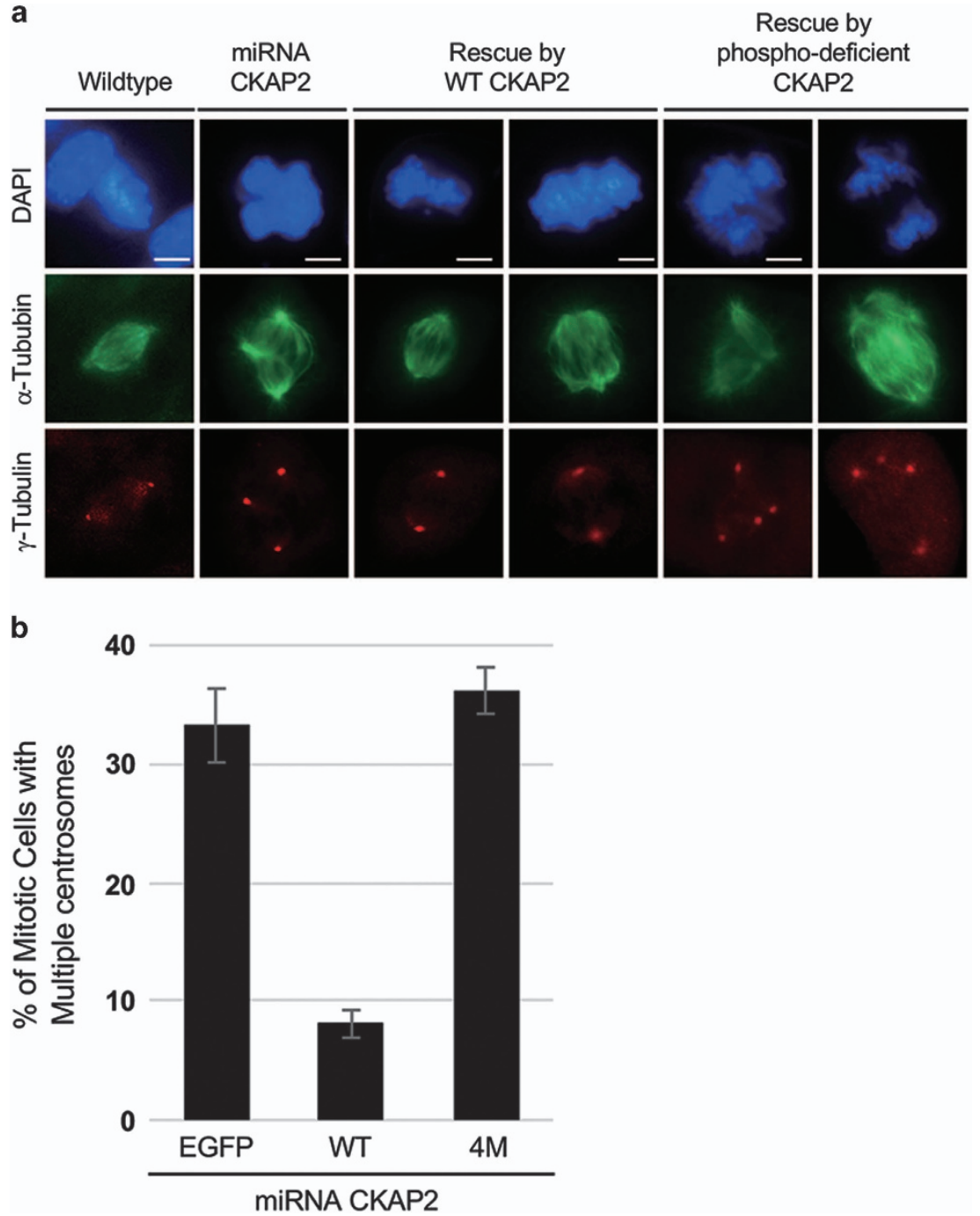

Figure 3 The mitosis-specific phosphorylation of CKAP2 T603 and S608 is related to centrosome integrity. (a) Lentiviral transduction with wild-type CKAP2 or phosphorylation-deficient CKAP2 was performed in CKAP2-depleted NIH3T3 cells that were co-immunostained with $\gamma$-tubulin (red) and $\alpha$-tubulin (green) and merged with DAPI (blue). Scale bar, $5 \mu \mathrm{m}$. (b) The quantification of cells with multiple $\gamma$-tubulin foci, presented as the mean values \pm s.e.m.

CKAP2 mutants with alanine at all four putative CDK1 phosphorylation sites could compensate for the effect of CKAP2 depletion. We introduced the phosphorylationdeficient mutants in CKAP2-depleted cells and compared their phonotype with that of wild-type CKAP2. Approximately $30 \%$ of mitotic cells, compared with wild-type cells, were found to have multiple $\gamma$-tubulin foci, thus indicating that the phosphorylation status of these putative CDK1 sites is crucial for the proper duplication of centrosomes (Figures $3 \mathrm{a}$ and $\mathrm{b}$ ). To determine which site failed to restore the centrosome number, we mutated each of these four residues to alanine. The introduction of CKAP2 with either the T561A or T579A mutation clearly restored the CKAP2 depletion effect, and two normal centrosomes were observed in mitotic cells (Figures $4 \mathrm{a}$ and $\mathrm{b}$ ). Therefore, the phosphorylation status of these two residues was not related to the maintenance of centrosome number. Interestingly, either T603A or S608A was still observed in multiple centrosomes in mitotic cells; thus, these mutations did not restore the CKAP2 depletion effect (Figures $4 \mathrm{a}$ and $\mathrm{b}$ ). These results suggested that the phosphorylation of T603 and S608 is essential for maintaining centrosome number.

CKAP2 T603 was phosphorylated by CDK1 in early mitosis In addition to in vitro phosphorylation, we investigated whether either T603 or S608 was phosphorylated in vivo. Using the previously reported human pT622 or pS627 antibody, ${ }^{14,15}$ we examined whether either T603 or S608 could be detected by these antibodies. Unfortunately, the anti-human-pS627 antibody did not recognize mouse pS608 (data not shown). However, the anti-human-pT622 (anti-pT603) antibody detected pT603, but it did not react with T603A mutants in the western blot analysis (Figure 5a). After cells were arrested at G2/M through treatment with nocodazole, the level of 


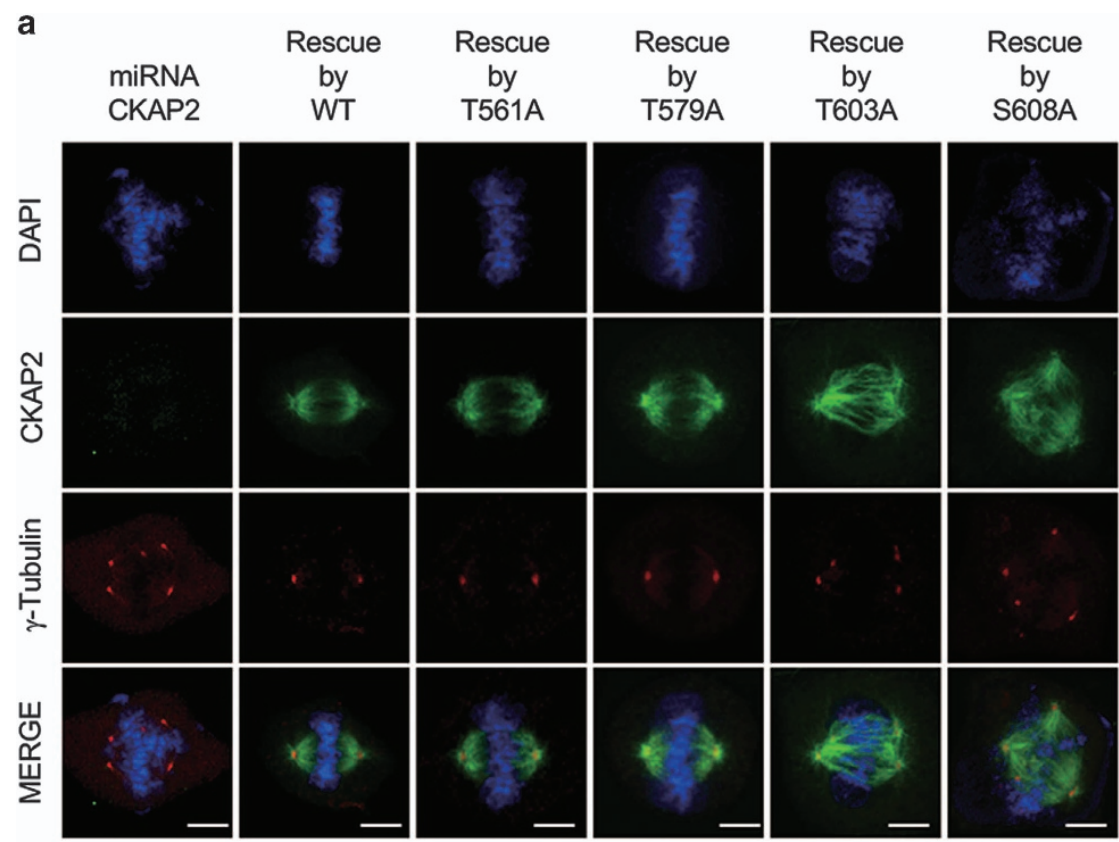

b

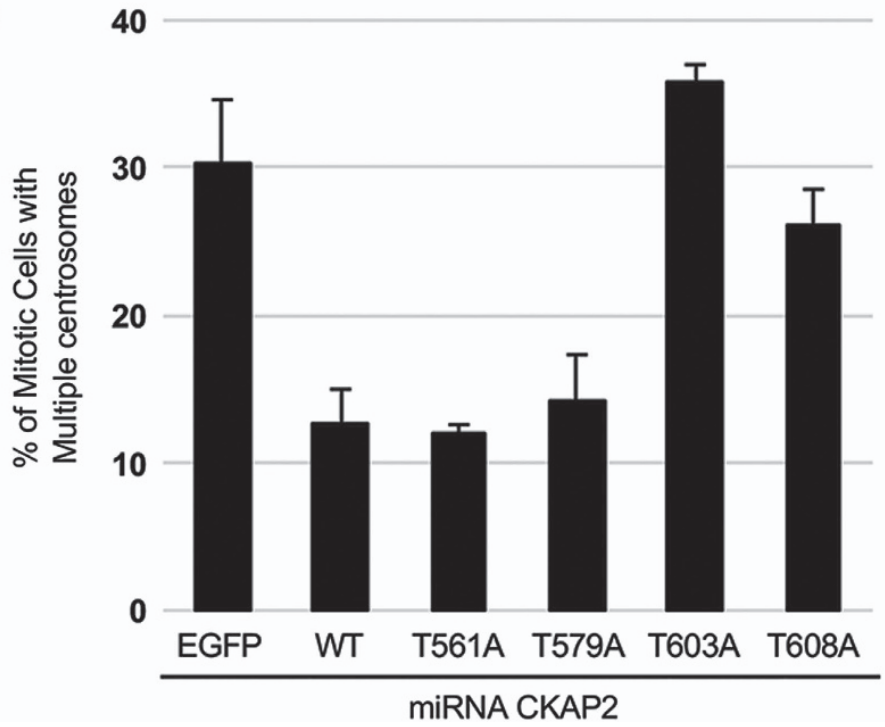

Figure 4 Mitosis-specific phosphorylation of CKAP2 T603 and S608 is related to centrosome integrity. (a) Lentiviral transduction with wild-type CKAP2 or indicated phosphorylation-deficient CKAP2 mutants was performed in CKAP2-depleted NIH3T3 cells that were co-immunostained with $\gamma$-tubulin (red) and CKAP2 (green) and merged with DAPI (blue). Scale bar, $5 \mu \mathrm{m}$. (b) The quantification of cells with multiple $\gamma$-tubulin foci presented as the mean values \pm s.e.m.

phosphorylated CKAP2 at T603 was greatly increased compared with that in asynchronized cells. However, this phosphorylation was almost completely absent after treatment with RO3306 (Figure 5b). We also examined when T603 was phosphorylated during the cell cycle phases. In interphase or cytokinesis, the CKAP2 proteins were not stained (Figure $5 \mathrm{c}$ ), but they exhibited strong staining on spindle poles and mitotic spindles during metaphase (Figure $5 \mathrm{c}$ ). Therefore, we concluded that at least T603 is phosphorylated in early mitosis. Together, our results suggest that the phosphorylation status at T603 and possibly S608 is essential for maintaining centrosome integrity.

\section{DISCUSSION}

The depletion of mouse CKAP2 has been reported to cause aberrant chromosome segregation. ${ }^{11}$ In addition, our recent study has shown that CKAP2 is responsible for centrosome integrity in primary hepatocytes. ${ }^{9}$ However, how CKAP2 contributes to maintain the number of centrosomes is unknown. Here we demonstrated that the phosphorylation status of CKAP2 is essential for maintaining the proper centrosome number.

To identify the phosphorylation sites of CKAP2 related to centrosome integrity, we used mass spectrometry with various fragmentation techniques and purified phosphopeptides by 

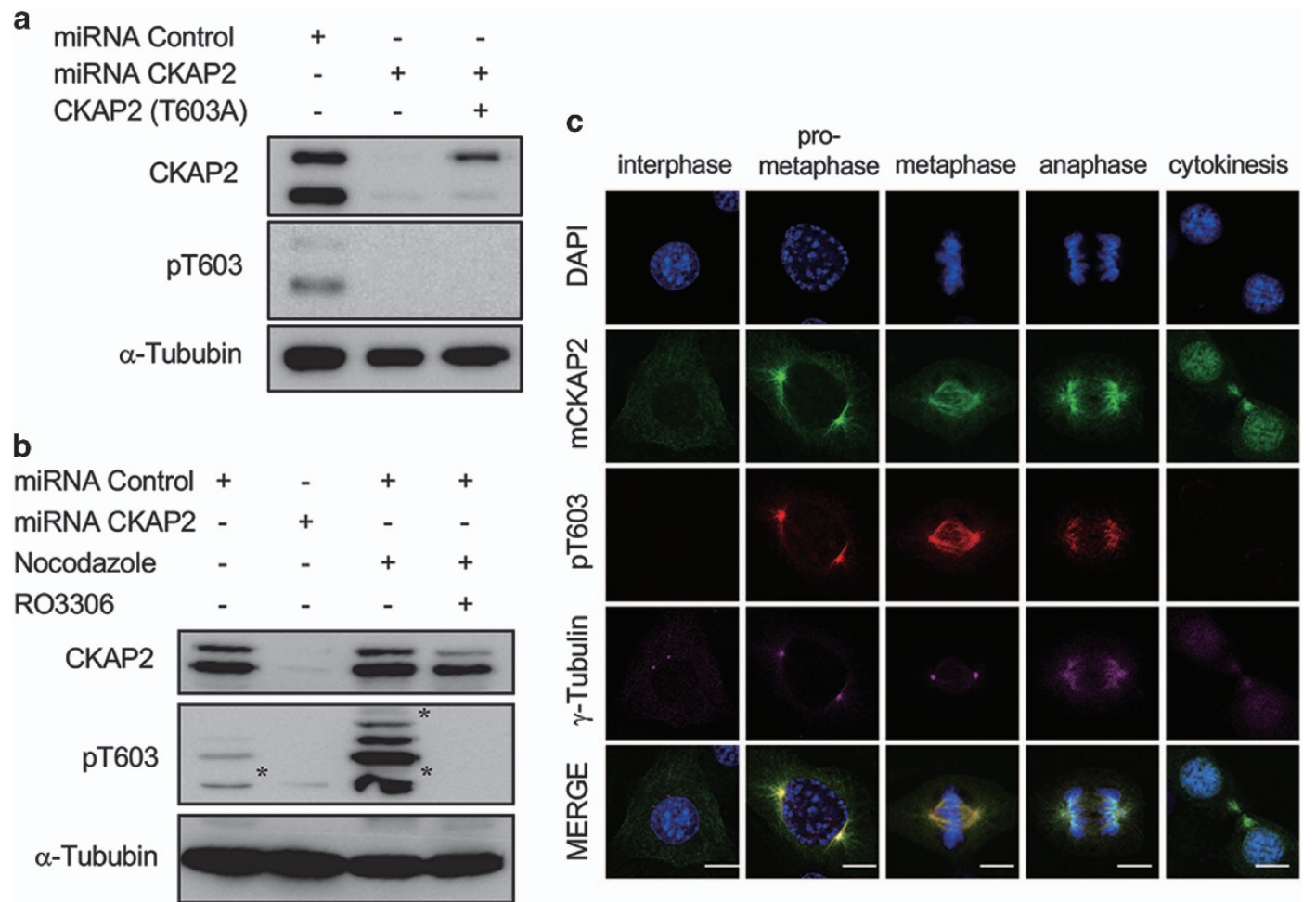

Figure 5 CKAP2 T603 was phosphorylated by CDK1 during early mitosis. (a) NIH3T3 cells were introduced to CKAP2 miRNA or the phosphorylation-deficient mutant and analyzed by western blotting. Western blot analysis showed that the phosphorylated T603-specific antibody specifically detected its epitope but could not detect the T603A mutant form of CKAP2. Non-specific bands are indicated with asterisks. (b) NIH3T3 cells G2/M arrested by nocodazole treatment were treated with DMSO or R03306 and analyzed by western blotting. Western blot analysis showed that the pT603 antibody strongly reacted with mitotic CKAP2 and was abrogated by R03306 treatment. (c) NIH3T3 cells were co-immunostained with CKAP2 (green), CKAP2 pT603 (red), and $\gamma$-tubulin (purple) and merged with DAPI (blue). Scale bar, $5 \mu \mathrm{m}$.

using the BioID system. Only the C-terminal region of human CKAP2 was phosphorylated in a mitosis-specific manner, ${ }^{14}$ and this region is well conserved between humans and mice. Therefore, we hypothesized that the phosphorylation sites in the $\mathrm{C}$ terminus of mouse CKAP2 may be involved in maintaining the number of centrosomes during mitosis.

As a result, six phosphorylation sites, S118, Y174, S216, S261, T561 and T579, were detected by mass spectrometry. Among them, T561 and T579 corresponded to mitosis-specific phosphorylation sites in human CKAP2. ${ }^{17}$ However, we were not able to analyze the region containing T603 and S608, which has been reported as the mitotic phosphorylation site in human CKAP2. This result might have been caused by multiple phosphorylation, a very low amount and a poor fragmentation in collision-induced dissociation, thereby resulting in poor sensitivity of the mass spectrometry technique. To verify this possibility, we performed an in vitro phosphorylation assay and analyzed the phosphorylation sites by mass spectrometry with electron transfer dissociation fragmentation. After in vitro phosphorylation using Cdk1/cyclin B1 or Aurora B/INCENP complex, we analyzed these phosphorylation sites by mass spectrometry with electron transfer dissociation and a kinase assay (Supplementary Figures $1 \mathrm{a}$ and $\mathrm{b}$ and Figures $2 \mathrm{e}$ and $\mathrm{f}$ ).

In our previous report, S627, corresponding to mouse CKAP2 S608, has been found to be phosphorylated by Aurora
B kinase in human CKAP2 ${ }^{15}$ but Aurora B kinase did not phosphorylate S608 of mouse CKAP2, as shown in an in vitro kinase assay (Supplementary Figure 1b). However, although S608 lacks proline in the +1 position and arginine in the +3 position $\left({ }^{608} \mathrm{SRRI}^{611}\right)$, in contrast to $\mathrm{T} 603\left({ }^{603} \mathrm{TPVR}^{606}\right)$, both residues were also phosphorylated by CDK1 in vitro. These results indicated that both $\mathrm{T} 603$ and $\mathrm{S} 608$ are phosphorylated by CDK1-cyclin B1. Therefore, we concluded that these four candidate phosphorylation sites (T561, T579, T603 and S608) have roles in centrosome integrity, and we further elucidated their roles by using phosphorylationdeficient CKAP2.

T603A and S608A, phosphorylation-deficient mutants of CKAP2, were not able to restore the abnormal number of centrosomes, whereas T561A and T579A were. These results were consistent with those of our previous studies of human CKAP2, which have shown that T622 and S627 are critical for the function of CKAP2 in controlling proper chromosome segregation and subcellular localization during mitosis, respectively. ${ }^{14,15}$ However, T578 and T596 mutations did not induce a significant delay during mitosis. ${ }^{17}$ Therefore, we focused on the phosphorylation sites of T603 and S608 in CKAP2. As shown in Figure 5, cells in early mitosis were stained with anti-pT603, but the phosphorylation of the residue was inhibited by a CDK1 inhibitor. These findings indicated 
that T603 was phosphorylated by CDK1 during mitosis. However, it is not clear whether S608 was phosphorylated by CDK1 in vivo, because of the lack of a specific antibody.

The mechanism by which T603A and S608A mutants destroy centrosome integrity is still unclear. One possibility is that phosphorylation at either T603 or S608 by CDK1 may trigger a confirmation change at its $\mathrm{C}$ terminus. This possibility is supported by one of our previous findings suggesting that the motifs surrounding S627 in human CKAP2 are important for both kinase recognition and subcellular recognition. ${ }^{15}$ Thus, the phosphorylation of $\mathrm{T} 603$ and $\mathrm{S} 608$ by $\mathrm{CDK} 1$ may contribute to maintaining centrosome integrity during the early phases of mitosis.

Together, these results strongly suggest that the phosphorylation of CKAP2 at T603 and possibly S608 by CDK1 during mitosis is essential for maintaining the number of centrosomes. Our present study provides a key clue as to the role of CKAP2 protein in maintaining centrosome integrity, and it should be highly significant for the development of anticancer targets.

\section{CONFLICT OF INTEREST}

The authors declare no conflict of interest.

\section{ACKNOWLEDGEMENTS}

This research was supported by a grant from the National R\&D Program for Cancer Control from the Ministry of Health and Welfare in the Republic of Korea (1120060).

1 Boveri T. Concerning the origin of malignant tumours by Theodor Boveri. Translated and annotated by Henry Harris. J Cell Sci 2008; 121(Suppl 1): $1-84$.

2 Montagna C, Andrechek ER, Padilla-Nash H, Muller WJ, Ried T. Centrosome abnormalities, recurring deletions of chromosome 4, and genomic amplification of HER2/neu define mouse mammary gland adenocarcinomas induced by mutant HER2/neu. Oncogene 2002; 21: 890-898.

3 Zhou H, Kuang J, Zhong L, Kuo WL, Gray JW, Sahin A et al. Tumour amplified kinase STK15/BTAK induces centrosome amplification, aneuploidy and transformation. Nat Genet 1998; 20: 189-193.

4 Schatten H, Wiedemeier AM, Taylor M, Lubahn DB, Greenberg NM, BeschWilliford $\mathrm{C}$ et al. Centrosome-centriole abnormalities are markers for abnormal cell divisions and cancer in the transgenic adenocarcinoma mouse prostate (TRAMP) model. Biol Cell 2000; 92: 331-340.

5 Jung CK, Jung JH, Lee KY, Kang CS, Kim M, Ko YH et al. Centrosome abnormalities in non-small cell lung cancer: correlations with DNA aneuploidy and expression of cell cycle regulatory proteins. Pathol Res Pract 2007; 203: 839-847.
6 Kellogg DR, Moritz M, Alberts BM. The centrosome and cellular organization. Annu Rev Biochem 1994; 63: 639-674.

7 Gergely F, Basto R. Multiple centrosomes: together they stand, divided they fall. Genes Dev 2008; 22: 2291-2296.

8 Chng WJ, Braggio E, Mulligan G, Bryant B, Remstein E, Valdez R et al. The centrosome index is a powerful prognostic marker in myeloma and identifies a cohort of patients that might benefit from aurora kinase inhibition. Blood 2008; 111: 1603-1609.

9 Yoo BH, Park CH, Kim HJ, Kang DS, Bae CD. CKAP2 is necessary to ensure the faithful spindle bipolarity in a dividing diploid hepatocyte. Biochem Biophys Res Commun 2016; 473: 886-893.

10 Bae CD, Sung YS, Jeon SM, Suh Y, Yang HK, Kim YI et al. Up-regulation of cytoskeletal-associated protein 2 in primary human gastric adenocarcinomas. J Cancer Res Clin Oncol 2003; 129: 621-630.

11 Hong KU, Kim E, Bae CD, Park J. TMAP/CKAP2 is essential for proper chromosome segregation. Cell Cycle 2009; 8: 314-324.

12 Jeon SM, Choi B, Hong KU, Kim E, Seong YS, Bae CD et al. A cytoskeletonassociated protein, TMAP/CKAP2, is involved in the proliferation of human foreskin fibroblasts. Biochem Biophys Res Commun 2006; 348: 222-228.

13 Hong KU, Park YS, Seong YS, Kang D, Bae CD, Park J. Functional importance of the anaphase-promoting complex-Cdh1-mediated degradation of TMAP/CKAP2 in regulation of spindle function and cytokinesis. Mol Cell Biol 2007; 27: 3667-3681.

14 Hong KU, Kim HJ, Kim HS, Seong YS, Hong KM, Bae CD et al. Cdk1-cyclin B1-mediated phosphorylation of tumor-associated microtubule-associated protein/cytoskeleton-associated protein 2 in mitosis. J Biol Chem 2009; 284: 16501-16512.

$15 \mathrm{Kim} \mathrm{HJ}$, Kwon HR, Bae CD, Park J, Hong KU. Specific primary sequence requirements for Aurora B kinase-mediated phosphorylation and subcellular localization of TMAP during mitosis. Cell Cycle 2010; 9: 2027-2036.

16 Roux KJ, Kim DI, Burke B. BiolD: a screen for protein-protein interactions. Curr Protoc Protein Sci 2013; 74: Unit 19.23.

17 Hong KU, Choi YB, Lee JH, Kim HJ, Kwon HR, Seong YS et al. Transient phosphorylation of tumor associated microtubule associated protein (TMAP)/cytoskeleton associated protein 2 (CKAP2) at Thr-596 during early phases of mitosis. Exp Mol Med 2008; 40: 377-386.

18 Prosser SL, Samant MD, Baxter JE, Morrison CG, Fry AM. Oscillation of $\mathrm{APC} / \mathrm{C}$ activity during cell cycle arrest promotes centrosome amplification. J Cell Sci 2012; 125: 5353-5368.

19 Hatano T, Sluder G. The interrelationship between APC/C and Plk1 activities in centriole disengagement. Biol Open 2012; 1: 1153-1160.

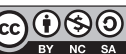

This work is licensed under a Creative Commons Attribution-NonCommercial-ShareAlike 4.0 International License. The images or other third party material in this article are included in the article's Creative Commons license, unless indicated otherwise in the credit line; if the material is not included under the Creative Commons license, users will need to obtain permission from the license holder to reproduce the material. To view a copy of this license, visit http:// creativecommons.org/licenses/by-nc-sa/4.0/

Supplementary Information accompanies the paper on Experimental \& Molecular Medicine website (http://www.nature.com/emm) 